\title{
Can Interactive Dual Fields of Information Explain the Prevalent Phenomena
}

\author{
Teruaki Ohnishi \\ Institute of Science and Technology for Society, Urayasu, Chiba, Japan
}

Email address:

ohnishiteruaki@yahoo.co.jp

\section{To cite this article:}

Teruaki Ohnishi. Can Interactive Dual Fields of Information Explain the Prevalent Phenomena. American Journal of Physics and Applications. Vol. 7, No. 6, 2019, pp. 144-155. doi: 10.11648/j.ajpa.20190706.12

Received: December 14, 2019; Accepted: December 26, 2019; Published: January 8, 2020

\begin{abstract}
The occurrence of prevalent phenomena is an almost unclear but interesting subject for us. Here we have constructed a dual model of information fields originated from the news media and showed that the quasi-cyclic appearance of prevalence can be explained by such a model. The homogeneous field of information around us was assumed, which is composed of the real field originated from the primary media such as newspapers and the television, and the cyber field from the PC and smart phones. The latter field is of the SNS cyber world affected by the field of real world. The public was assumed to be influenced simultaneously by these two types of fields to result in the enhancement of the awareness of some specific things. To investigate the viability of such a dual model, inputting the data of the real field regarding the global warming (GW) already reported in Japan as an external variable, the feature was derived in what manner the public awareness of GW had varied during the past $\sim 35$ years. The high public awareness was found to be realized at around 2009 when the information environment was explosively enhanced in the real world. Such enhancement of the awareness could be explained by the contribution from the cyber field, which was brought by the instability of the field, or a burst, induced by a small perturbation from the real field. A possibility was pointed out that the spontaneous occurrence of quasi-cyclic instability such as the case of our explosive awareness could take place in the interactive dual system of information between the real and cyber fields. We pointed out that the spontaneous occurrence of prevalence in general could be explained also by the similar mechanism as ours.
\end{abstract}

Keywords: Duality of Information Fields, Quasi-cyclic Prevalence, Public Awareness, SNS, News Media, Global Warming, Burst, Real and Cyber Worlds

\section{Introduction}

Major news sources in 2018 in Europe (UK, France and Germany) are the television (over 70\%), online media $(70 \sim 75 \%)$, social media $(\sim 40 \%)$, printed newspapers (20 35\%) and so on [1]. On the other hand in USA, they are respectively $49 \%, 33 \%, 20 \%, 16 \%$ and so on [2]. Both in Europe and USA, the social media becomes the most important means in the acquisition of everyday news. Also in Japan the reading rate of net news $(71 \%)$ becomes surmounted over the rate of printed newspapers (69\%) [3], and moreover the using rate of the social media broadly distributes from $29 \%$ of the people of 70 s years old to $77 \%$ of teenagers [4]. For the social media, the rate of retrieval of news information is almost comparable to that rate of topical and worldly information [4]. Thus at present, the primary information offered by the television, newspapers and online news, and the secondary information from the cyber world on SNS form the information environment in the society, being fused and mutually intermingled.

As such information from the media reaches the people with different characteristics from each other, different awareness is induced in each people with every matters of news. This corresponds to the microscopic awareness or the microscopic opinion so that its averaging behavior in a whole society becomes the macroscopic awareness of the society [5-7]. On the other hand, when we macroscopically treat the social group without considering each people by assuming the average quantities of the group just as the collective characteristics of the group [8,9], we directly obtain the macroscopic awareness and public opinion of that social group. In the field of social physics, many studies have been done regarding the change of microscopic cognition under the influence of news media [10-15]. As for the latter macroscopic 
treatment, there also exist many investigations [16-20]. Ohnishi and Shimano [21] considered the social environment formed by the information released from the news media from time to time. They assumed the environment homogeneously prevailing throughout the whole society, forming a sort of field, the information filed. They also assumed the people immersed in the time-varying field and equilibrated with the field, which changed the state of awareness and attitude according to the atmosphere of the field. In this case, however, a homogeneous field is of the field averaged over the information originated from various types of media only in the real world, and the cyber field which is a field formed in the social network service (SNS) in the cyber world is not considered.

It is well known at present that the SNS communication between a large number of general public induces the temporary or periodic occurrence of a strong cyber field in the cyber world as a result of the intensive exchange of vast amount of information between people at one time [22, 23] In the cyber world, the expansion of information is also known to occur rapidly throughout a quite wide region of society by the network communication even by little supply of information [24-28]. In this case the awareness and attitude of the public are apt to be assembled toward a certain biased direction. Thus the public awareness and attitude can not be considered without taking the cyber field into account, and hence the dual model of information environment of real and cyber worlds is now necessarily required in treating the public awareness and attitude to every things.

Granell et al. [29] introduced a dual model with different networks of real world and SNS cyber world from each other though they correspond to a single society with a single member of people. When the direct contact between people is restricted in the real world owing to the exchange of information in the cyber world, the introduction of such a model seems to be effective for preventing the expansion of epidemics in case of their prevalence. Improving such a type of dual model, Wang et al. [39], Dong et al. [31], Yi et al. [32], and Wang et al. [33] have introduced dual type models where agents have different characteristics in the two worlds from each other to discuss the diffusion of epidemics and rumors. Although in these models the topological structure of the network was assumed unchanged with time, Lee et al. [34] assumed in their epidemic model the characteristics of agents changing with the propagation of epidemics, which induce the change of network structure, that is the topology in the real world. Their model, however, is not a model of network with a duality.

To derive the microscopic awareness and/or attitude of people immersed both in the real and cyber fields, such a dual model with different characteristics of agents in the two different worlds may be effective. To discuss the macroscopic features throughout the society considered, however, it may be difficult to directly apply such a type of dual model using agents.

In this paper the duality of the real and cyber worlds is in general considered, without restricting the subject only to the epidemic or rumor expansion, to calculate the macroscopic and collective awareness and/or attitude of people by taking into account the real and cyber fields of information. In the next section the prerequisites for our model is shown, and the formulation is made in Section 3. In Section 4, for the verification of this model, a problem is exemplified how the Japanese have changed the awareness and attitude to the global warming $(\mathrm{GW})$, where we show the calculation well explainable for the behavior of observation. Moreover we point out the possibility that a small perturbation in the real world induces instability in the cyber world to result in a temporary change of collective awareness to a greater extent. Also pointed out is the possible explanation of prevalent phenomena in general by our model. The conclusion is made in Section 5.

\section{Model}

In this paper we investigate in what manner the collective awareness of the public or the public interest in a certain social matter vary with the change of information environment prevailing in the society. This corresponds to the problems how the public opinion regarding, for instance, elections, environmental problems, or gun terrorism can be related to the amount of news which report those matters [35]. Here we set the following prerequisites.

1) The primary information regarding a certain subject is released in one direction from the media such as the (printed or online) newspapers, the television, or magazines to the society. The diffusion of information is made not only by the every day communication between people in their communities, working places or schools together with the direct reach from the media to the public, but also by the indirect communication in the cyber world. When the information widely diffuses throughout the society, the public may feel as to be surrounded by a sort of atmosphere or a sort of field, that is, the information field. Such a field smoothly prevails throughout the social space to influence on the awareness and attitude of the public. We can imagine that the public lives in such a field in equilibrating with it in a sense. In this case the field is assumed to be spatially homogeneous and how the public react to the field depends on the characteristics of each people.

2) The real field is formed by the information field in the real world, whereas the cyber field is formed in a cyber space when the public intends to transmit and distribute information by means of the Internet and SNS such as Twitter, Instagram, or Facebook which are the communication tools laying emphasis on sending, prevailing and jointly possessing information. If such a cyber field is also homogeneous in the society, the public is imagined to change his awareness and attitude to every subject, being simultaneously influenced by those fields of dual type.

3) After news is released at first from the news media and it causes some interest within someone, he sends it through the cyber network just as it is or by transforming and 
changing somewhere as he wants to intend. In the latter case the main point at issue or the point of view may be different from those in the real world since they are reflected the interests or the attitude of a person who send the cyber message [36]. Thus, the cyber field can introduce the information in the real field together with the cognitive state of the public. Although the influence from the real to the cyber worlds will be modeled as such, that in the reverse direction and the public's cognitive effect are not taken into account in our model.

4) According to these images, the awareness and attitude of the public are influenced, separately but at the same time, from the dual fields of real and cyber ones. As the news release from the news media is usually repeated in the real world, the above processes from 1) to 3) are also repeated every time. Figure 1 shows a schematic diagram of such a system. Here the primary information in the real world is the external phenomenon that randomly occurs in time. In this figure, the environmental field is assumed to make the extent of the public's knowledge and understanding enhanced and, through the enhancement, the public's awareness is also assumed enhanced [37]. Moreover the enhancement of the public's awareness is tacitly assumed to elevate the social responsibility regarding that problem, furthermore leading the information seeking about the subject [37, 38]. Moreover the oblivion is introduced which makes the extent of knowledge and understanding to have a maximum and saturated value.

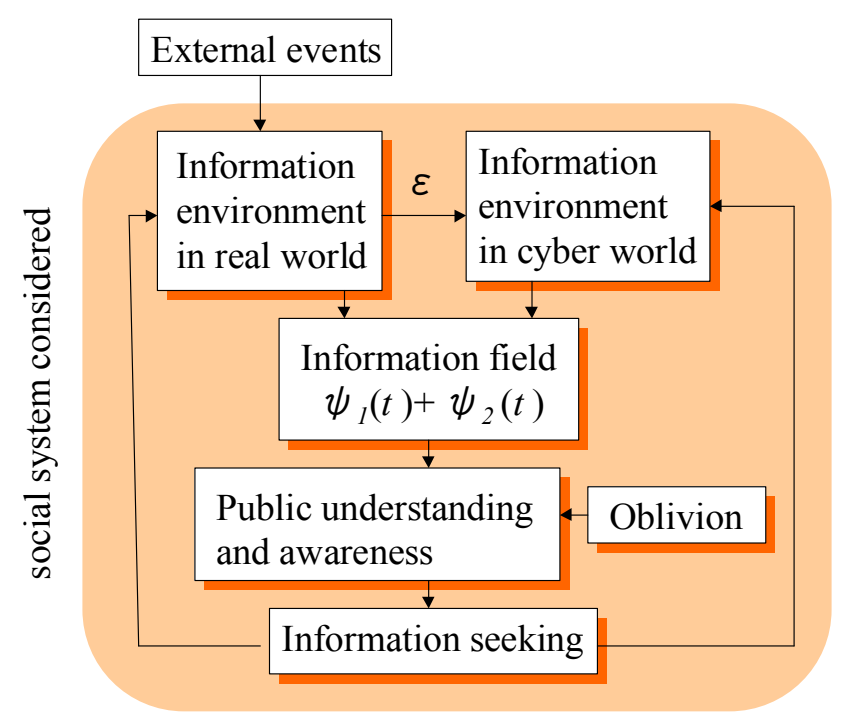

Figure 1. Schematic diagram of our model.

In the next section we make a formulation for such a model.

\section{Formulation}

\subsection{Basic Relation}

We introduce the information fields according to Figure 1, which consist from the real and cyber fields, $\Psi_{1}$ and $\Psi_{2}$, respectively. The real field with respect to a certain subject $\omega$ is the sum of the information environment formed by newspapers, the television, magazines and other primary sources, and the environment of social atmosphere formed by the public-relations activities by the government, NGOs or private enterprises and by the education at school and the communication in communities. The subject $\omega$ tends to be repeatedly reported by the news media if the $\omega$ is really an important subject the public pay attention to. Hence the real field $\Psi_{1}$ is a function of time $t$, the average of the public's awareness $\langle\xi\rangle$, and $\left.\omega, \Psi_{1}(t,<\xi\rangle, \omega\right)$. We call the $<\xi>$ as "awareness" for simplicity hereafter.

The source of the information that distributes in the cyber world is assumed to originate from the real world. The information thus input in the cyber world is repeatedly sent and received within the cyber world, or in other words fed back, through the cyber network. When its feedback is positive, the field $\Psi_{2}$ becomes increased, whereas it rapidly deceases and the $\omega$ never becomes the talk when the feedback is negative. Whether the $\omega$ becomes a topic in the cyber world depends on the extent of public awareness $\langle\xi\rangle$, which is approximately proportional to the number ratio of the people who are aware of $\omega$ to the total. The amount of information sent and received in the network depends on the number of effective nodes of the network, which may be proportional to the $q^{\prime}$ th powers $(q>1)$ of the number ratio of the people who are aware of $\omega$ to the total. Hence the field $\Psi_{2}$ in the cyber world is given in the following form.

$$
\psi_{2}(t+\Delta t)=\eta \cdot\langle\xi\rangle^{q}\left\{\psi_{2}(t)+\varepsilon \psi_{1}(t)\right\}
$$

where $\varepsilon(<<1)$ is a constant which represents the extent for the cyber network to introduce the information in the real world, $\eta$ is a constant depending on the $\omega$ and the macroscopic character of the society considered, and $\Delta t$ is a small time span corresponding to the feedback time. When the feedback value becomes greater than 1.0, $\Psi_{2}$ increases every time after the elapse of time $\Delta t$, rapidly growing within a small time (the situation is discussed afterward). The strength of the field regarding $\omega$ at a time $t$ is

$$
\psi(t)=\psi_{1}(t)+\psi_{2}(t)
$$

In the next place we consider the expression $\langle\xi\rangle$. The quantity $\xi \in[0,1]$ is a variable giving the extent of public awareness regarding the subject considered, and $\xi=0$ and 1 correspond respectively to the people without any interest and the people with maximum saturated interest. Taking into account the whole society, we set $\Xi \cdot d \xi \equiv \Xi(\xi, t, \Omega) \cdot d \xi$ as the fraction of people who possesses the awareness within $[\xi$, $\xi+d \xi$, where $\Omega$ is a variable totally including the subject $\omega$ and the demographic characteristics of the public such as the age, the gender or the occupation. In what follows we only consider, for simplicity, the collective behavior of people who have the characteristics averaged over the society, assuming the $\Xi$ being a function of only $t$ and $\xi$.

The variation of $\Xi$ within an interval $[\xi, \xi+d \xi]$ during a time span $[t, t+d t], \Xi \cdot d \xi d t \equiv \Xi \cdot d \Xi$, is given by the sum of the 
amounts of inflow and outflow toward the right hand side, $R_{+} d t$ and $R \_d t$, that corresponds to the rising direction of awareness, whereas the amount of inflow and outflow toward the left hand side, $L_{+} d t, L \_d t$, corresponding to the falling of the awareness as shown in Figure 2.

$$
d \Xi=\left(R_{+}+L_{+}\right) d t-\left(R_{-}+L_{-}\right) d t
$$

Here we suppose that the public awareness increases approximately in proportion to the amount of information supplied during a unit time, that is, the strength of the information field. Such proportionality, however, may not be held well throughout the whole region in $\xi \in[0,1]$. This is because the increasing rate of $\Xi$ becomes not to depend so strong on the field with the approach of $\xi=1$ where people scarcely vary their $\xi$ due to its saturation. The variation towards the right direction, $\left(R_{+}-R-\right) d t$ is then given by

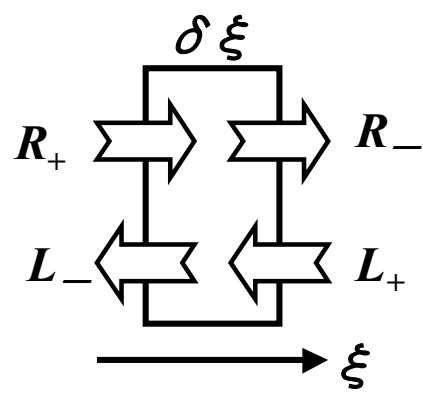

Figure 2. Definition of inflows and outflows in the $\xi$-direction.

$$
\begin{aligned}
\left(R_{+}-R_{-}\right) d t & =\psi(t) \cdot\left\{a\left(\xi^{\prime}\right) \Xi\left(\xi^{\prime}\right)-a(\xi) \Xi(\xi)\right\} \cdot d t \\
& =\psi(t) \cdot \frac{a\left(\xi^{\prime}\right) \Xi\left(\xi^{\prime}\right)-a(\xi) \Xi(\xi)}{\xi^{\prime}-\xi} \cdot \delta \xi d t \quad\left(\xi^{\prime}-\xi=\delta \xi<0\right) \\
& =\psi(t) \cdot \frac{d\{a(\xi) \Xi(\xi)\}}{d \xi} \cdot d \xi d t \quad(\delta \xi \rightarrow 0) \\
& =a(\xi) \cdot \psi(t) \frac{d \Xi(\xi)}{d \xi} \cdot d \xi d t+c(\xi) \cdot \psi(t) \cdot \Xi(\xi) d \xi d t
\end{aligned}
$$

where $a(\xi)$ is a factor to revise the variation of the awareness, which is assumed to be proportional to the information field $\Psi$, towards a realistic variation (which will be considered later), $c(\xi) \equiv d a(\xi) / d \xi$, and $\xi^{\prime}=\xi-\delta \xi$, and note the above (4) is an expression in a limited condition of $\delta \xi \rightarrow 0$.

On the other hand, $L_{+} d t$ is the fraction of the component $[\xi$, 1] which falls into the region $[\xi, \xi+\delta \xi]$ during $[t, t+d t]$, and is given by

$$
L_{+} d t=\int_{\xi^{\prime}=\xi}^{1.0} \lambda_{2}\left(\xi, \xi^{\prime}\right) \cdot \lambda_{1}\left(\xi^{\prime}\right) \Xi\left(\xi^{\prime}\right) \Phi(t+d t, t) d \xi^{\prime} d \xi d t
$$

where $\Phi(t+d t, t)$ is the forgetting probability (or the oblivion function) of knowledge during $[t, t+d t]$. We have tacitly assumed that the loss of the awareness is fundamentally the same phenomenon as the oblivion of knowledge. Generally speaking, people who possesses a large $\xi$ do not lose knowledge so easily because of their strong memory due to the strong awareness, whereas people with a small $\xi$ apt to swiftly forget it due to the weak awareness. Thus the extent of oblivion depends on $\xi$, and the factor $\lambda_{l}(\xi)$ represents such dependency on $\xi$. Moreover $\lambda_{2}\left(\xi, \xi^{\prime}\right)$ is the falling fraction of $\Xi$ from $\left[\xi^{\prime}, \xi^{\prime}+d \xi^{\prime}\right]$ to $[\xi, \xi+d \xi]$ by the oblivion. These factors will be considered also in the next subsection.

The $L-d t$ is simply given by

$$
L_{-} d t=\lambda_{1}(\xi) \Xi(\xi) \Phi(t+d t, t) d \xi d t
$$

We finally obtain an expression for $\Xi \cdot d \xi d t \equiv d \Xi$ by totaling the above all as

$$
\begin{aligned}
d \Xi(\xi, t) & =\left(R_{+}+L_{+}\right) d t-\left(R_{-}+L_{-}\right) d t \\
& =\left[a(\xi) \psi(t) \frac{d \Xi(\xi)}{d \xi}+c(\xi) \psi(t) \Xi(\xi)+\Phi(t+d t, t)\left\{e(\xi)-\lambda_{1}(\xi) \Xi(\xi)\right\}\right] d \xi d t
\end{aligned}
$$

or

$$
\frac{\partial^{2} \Xi(\xi, t)}{\partial \xi \partial t}=\alpha(\xi, t) \frac{\partial \Xi(\xi, t)}{\partial \xi}+\beta(\xi, t) \Xi(\xi, t)+\gamma(\xi, t)
$$

where

$$
e(\xi) \equiv \int_{\xi^{\prime}=\xi^{\prime}}^{1.0} \lambda_{2}\left(\xi, \xi^{\prime}\right) \lambda_{1}\left(\xi^{\prime}\right) \Xi\left(\xi^{\prime}\right) d \xi^{\prime}
$$

and $\alpha, \beta$ and $\gamma$ are

$$
\begin{gathered}
\alpha(\xi, t)=a(\xi) \psi(t) \\
\beta(\xi, t)=c(\xi) \psi(t)-\lambda_{1}(\xi) \Phi(t+d t, t) \\
\gamma(\xi, t)=e(\xi) \Phi(t+d t, t)
\end{gathered}
$$

By using the distribution function $\Xi$, the $\langle\xi\rangle$ is given by

$$
\langle\xi\rangle \equiv\langle\xi(t)\rangle=\int_{0}^{1} \xi \Xi d \xi / \int_{0}^{1} \Xi d \xi
$$


In the next place we consider the constants and factors above cited.

\subsection{Auxiliary Equations}

\subsubsection{Factor $a(\xi)$}

This factor gives the extent in what manner the public's trend to seek information increases with $\xi$ when they are immersed in the information field. The extent that the people seek the information about a certain subject grows with the amount of knowledge of their own [37, 38]. When the knowledge becomes to be saturated, however, the seeking trend gradually decreases, and it must fall to nearly null in a limit of $\xi=1$. As for a function $A(\xi)$ that is approximately proportional to $\xi$ when $\xi<\xi_{m}$, grows maximum at $\xi=\xi_{m}$, and becomes null at $\xi=1.0$, we adopt

$$
A(\xi)=n(1-\xi) \cdot \exp (1-n+n \xi)
$$

where $n$ is a parameter. The above equation is normalized so as to become a maximum 1.0 within $\xi \in[0,1]$. By using this factor the quantity $a(\xi)$ is

$$
a(\xi)=a_{0} A(\xi)
$$

where $a_{0}$ is a constant. The function $c(\xi)$ in (11) is then given by

$$
c(\xi) \equiv d a(\xi) / d \xi=a_{0} \cdot n(n-n \xi-1) \cdot \exp (1-n+n \xi)
$$

\subsubsection{Oblivion Function $\Phi(t+d t, t)$}

Although many studies have been done about how the memory possessed at a time $t+d t$ by someone, which is originally obtained at $t$, is expressed as a function of $d t$, it is unclear even now. Here we introduce the following formula [39] for such an oblivion function that decreases with $d t$ with a power law, but the form of its dependency on time does not essentially influence on our results.

$$
\phi(t+d t, t)=g\left(\frac{t_{0}}{t_{0}+d t}\right)^{\ell}+(1-g)
$$

where $\ell$ is a constant, $t_{0}$ is a parameter, and $g$ is the fraction of memory finally disappears at an infinite time. Hence our forgetting fraction is given by $\Phi \equiv(1-\varphi)$. When $d t<<1$, the $\Phi(t+d t, t)$ in (7), (11) and (12) is given by

$$
\Phi(t+d t, t)=\frac{g \ell}{t_{0}} d t
$$

\subsubsection{Inhomogeneous Oblivion Factor $\lambda_{1}(\xi)$}

The loss of memory with time does not take place homogeneously throughout the society, since a person who possesses a large $\xi$ does not easily forget his memory but a person who does not so may forget it promptly. The factor $\lambda_{I}(\xi)$ in (7) and (11) is a function that gives such an inhomogeneous oblivion effect. Here we assume the following functional form

$$
\lambda_{1}(\xi)=\exp \left(-\lambda_{1}^{0} \xi^{q_{1}}\right)
$$

where $\lambda_{l}{ }^{0}$ and $q_{1}$ are constants.

\subsubsection{Oblivion Distribution Factor $\lambda_{2}(\xi, \xi)$}

When a person losses his awareness during $\Delta t$ from $\xi$ to $\xi$, the resultant $\xi^{\prime}$ under a fixed $\xi, \lambda\left(\xi^{\prime}, \xi\right) \equiv \lambda_{2}\left(\xi^{\prime}, \xi\right) \cdot \lambda_{I}(\xi)$, does not homogeneously distribute over $\xi$ ' because the distribution near $\xi$ may relatively dense whereas it may become thin in the case of a large $\left(\xi-\xi^{\prime}\right)$. Namely when $\Delta t<<1$, the fraction of people who largely losses the awareness during $\Delta t$ will be small so that the $\lambda\left(\xi^{\prime}, \xi\right)$ may have a form with a larger weight near $\xi$. Although in this case the function $\lambda_{2}\left(\xi^{\prime}, \xi\right)$ works as the factor that gives such an effect, we know nothing about its functional form. Here we introduce the following form

$$
\lambda_{2}\left(\xi^{\prime}, \xi\right)=\exp \left\{-\lambda_{2}^{0}\left(\xi-\xi^{\prime}\right)^{q_{2}}\right\}
$$

where $\lambda_{2}{ }^{0}$ and $q_{2}$ are constant.

\subsubsection{Boundary and Initial Conditions for Equation (8)}

Equation (8) is rewritten as the simultaneous partial differential equations with two unknowns as

$$
\begin{gathered}
\partial \Xi / \partial \xi=X \\
\partial X / \partial t=\alpha(\xi, t) X+\beta(\xi, t) \Xi+\gamma(\xi, t)
\end{gathered}
$$

Of these two, we impose the following boundary conditions on $(22)$

$$
\begin{gathered}
\partial X /\left.\partial t\right|_{\xi=0}=\left(g_{0} \ell / t_{0}\right) \cdot e(0) \cdot \Delta \xi-\psi(t) a(0) \Xi(0) \\
\partial X /\left.\partial t\right|_{\xi=1}=\psi(t) a(1) \Xi(1)-\left(g_{0} \ell / t_{0}\right) \cdot \lambda_{1}(1) \cdot \Xi(1) \Delta \xi
\end{gathered}
$$

where $\Delta \xi$ is a discrete unit in the direction of $\xi$.

Moreover for the following numerical calculation, we set the following normal distribution as an initial condition for $\Xi(\xi, t)$ at $t=0$

$$
\Xi(\xi, 0)=N \cdot \exp \left\{-\left(\xi-\xi_{0}\right)^{2} /(2 \sigma)^{2}\right\}
$$

where $N$ is a normalization constant, and $\sigma$ is a constant. The functional form for the initial distribution and the values of parameters influence only negligibly on the results after a sufficiently long time from the beginning of calculation.

\section{Conditions for Numerical Calculation}

We investigate in what follows whether our model can explain Japanese awareness to the problem of global warming (GW) in the past. The same problem was already studied with a model different from the one described here [21], where no consideration was made on the transmission and dispersion of information on the cyber network. We study here to show how the cyber field varies in case of external news supply and in what manner the public awareness varies with the field. 


\subsection{Amount of Information on $G W$ by the News Media and the Public Awareness in Japan}

Figure 3(a)-(c) show the secular variation of the number of articles or programs on GW reported per month since $\sim 1990$ in Japan. These are the primary information that should be distinguished from the secondary one distributed via the cyber network such as SNS. Figure 3(a) is the distribution of the articles by three representative newspapers in Japan, the Mainichi, the Asahi and the Yomiuri, which can be retrieved from data bases with the keywords (global warming $U$ midsummer day) in Japanese. This is an average value for those newspapers with the weight of their respective circulations [21]. Figure 3(b) is the number of TV program broadcasted by NHK in the whole country [40], and (c) is the number of articles appeared in weekly and monthly magazines [41], whereas, though it is not a reportorial amount of news, (d) shows the number of search of internet articles using the Google search engine [42], all of these being retrieved by a keyword global warming in Japanese.
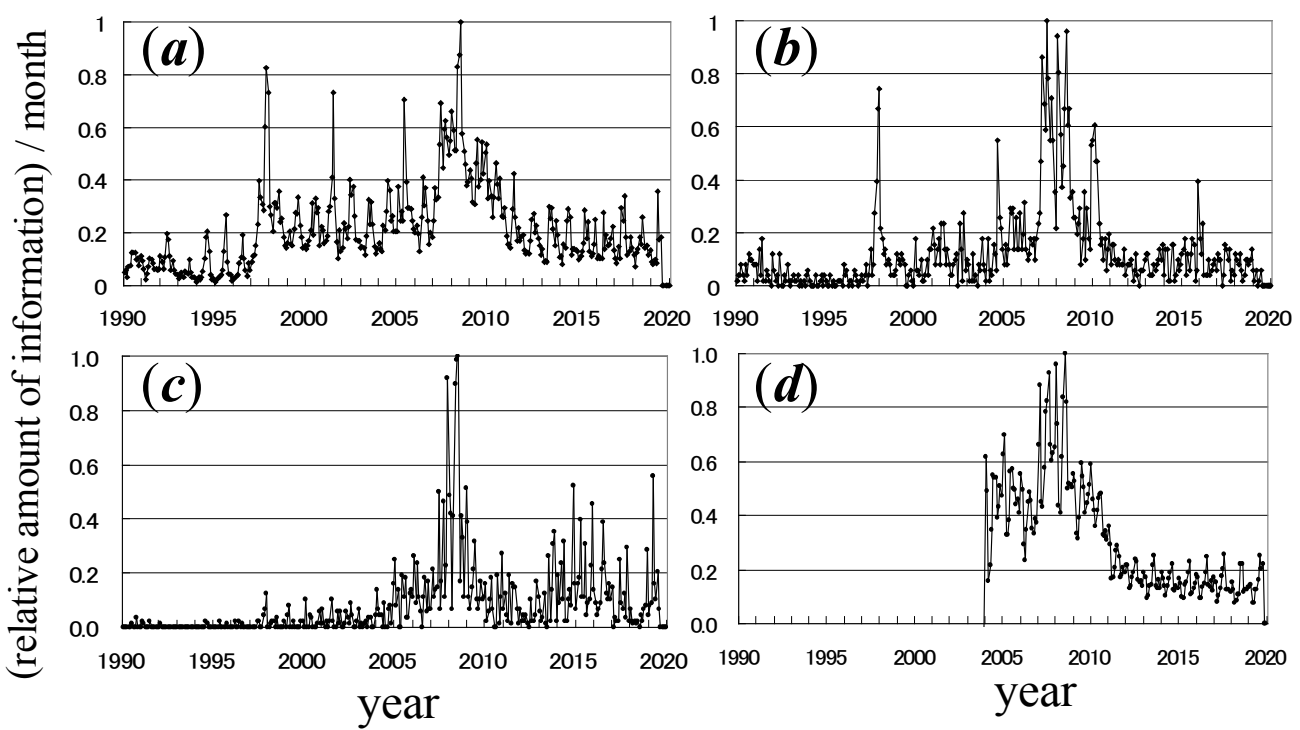

Figure 3. Amount of information per month on GW normalized each maximum as 1.0. (a) Number of articles in printed newspapers, (b) number of programs by the television, (c) number of articles in monthly and weekly magazines, and (d) number of retrieval on Google portal site only after 2004.

These figures (a)-(d) show a similar distribution to each other, having a common maximum at around 2008 2009, indicating that the information field regarding $\mathrm{GW}$ was explosively strengthened, or burst throughout the society at around that time. The reportorial peak appeared at December 1997 is due to Kyoto Conference on the Prevention of Global Warming, whereas the peak at 2007 2008 to COP13 Paris Conference where was adopted the Road Map for reducing the greenhouse gases, Lake Toya Summit in Hokkaido, Japan with a main subject of Global Environment, and the enhancement of public interest in natural energy sources.

\subsection{Setting Values of Constants}

A driving force of our model is the observed values shown in Figure 3(a)-(c). We introduce the following quantity $\Psi_{l}(t)$ as the input for the model

$$
\psi_{1}(t)=\sum_{i=1}^{3} c_{i} \varphi_{i}
$$

where $c_{i}$ is the weight for the new media $i(i=1,2,3$ for newspapers, the television and magazines, respectively), which we set from a public opinion poll [44] as $c_{1}=0.334$, $c_{2}=0.522$, and $c_{3}=0.104$. We do not take into account the news media other than these three types, excluding, for simplicity, the secondary news sources in the real world such as the communication in communities and the education at schools or by means of public relations. The form of $\Psi_{l}(\mathrm{t})$ is shown in Figure 4.

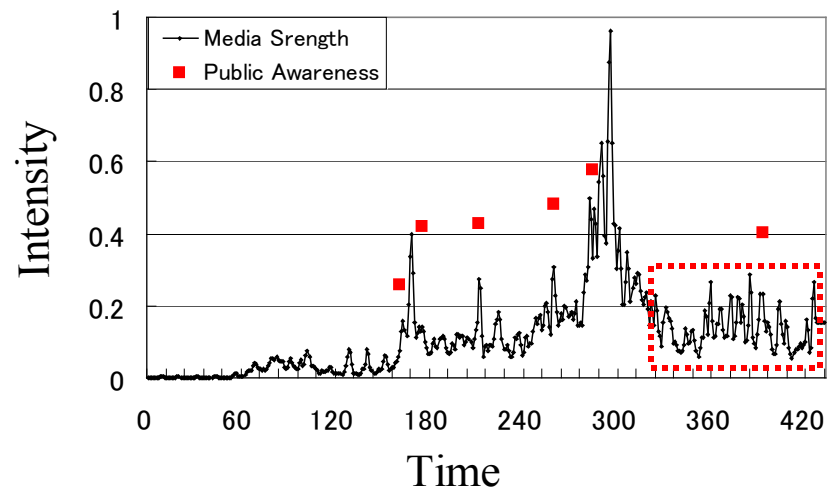

Figure 4. Quantity $\Psi_{l}(t)$ to be input into our model (lines) and the observed value of Japanese awareness to $G W$ (red squares). The region to be repeatedly used for the calculation of Figure 9(a) is indicated by the red dotted rectangle. Although the origin of time is set at the beginning of 1984, the abscissa is assumed to be of the time without any unit of time for general discussion.

Also in Figure 4, the rates of people who have an interest in GW are shown at several observational times [21]. Those are the values averaged for all subjects of public surveys who responded yes to the question such as "are you interested in the problems on global warming and the global environment?" There exist only five observations shown there where the 
same question in the same context as each other was made. The rates of people within subdivided demographics regarding the age, the gender or the occupation show the similar time trend as Figure 4 though the absolute values differ from each other. There is no observations around the time 2008 2009 when the $\Psi_{l}(\mathrm{t})$ became maximum, but in August 2007. The behavioral trend of those observations with time seems to be similar to that of $\Psi_{l}(\mathrm{t})$, indicating the public awareness affected, more or less, by the information field formed by the news media. It is our model that links the $\Psi_{I}(\mathrm{t})$ and the rate of people who has the interest in GW. We set no units of time in the abscissa in Figure 4, so that we investigate the response of a social system in general when an external input varies with time as given in Figure 4 with an arbitral unit of time.

We set the values of constants appeared in Section 3 as follows, but their values do not essentially influence on our results except for $a_{0}, t_{0}$ and $\eta ; q=2, q_{1}=q_{2}=1, n=5, g=1, \ell=2$, $\lambda_{1}{ }^{0}=1, \lambda_{2}{ }^{0}=1, \varepsilon=0.0001, \sigma=0.2, \xi_{0}=(0.75+0.5) / 2=0.625$. The quantities $a_{0}, t_{0}$ and $\eta$ are treated as parameters varying respectively in the ranges $a_{0} \in[18,34], t_{0} \in[10,18]$ and $\eta \in[2,4]$.

We differentiate (21) and (22) and numerically calculate them with $\Delta t=1 / 10$ and $\Delta \xi=1 / 80$. In this case the cyber field is fed back with every $\Delta t$ according to (1), indicating the information of that field updated in every $\Delta t$.

The extent of the quantities in real world, for instance, such as the amount of information and the number of people, is in general finite. Even if the amount of information grows large in the cyber world due to a positive feedback, it never expand infinitely but to a finite extent. Hence we set the strength of the

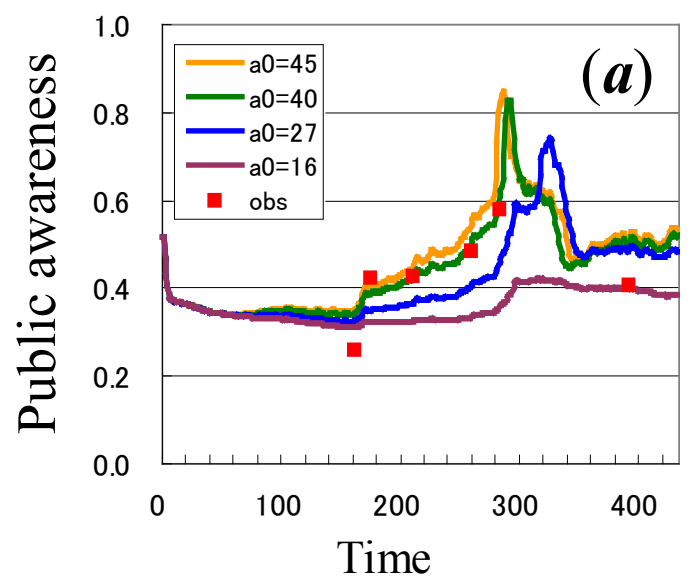

cyber field $\Psi_{2}(t)$ within a range $\Psi_{2}(t) \in\left[0, v \Psi_{1}(t)\right]$, where $v$ is a constant parameter.

King et al. [45] studied the public awareness about especially adjusted topics on the immigration, the climate and the educational policy by using Twitter after they released those articles through the online news media. They analyzed over 13000 Twitters from over 7000 people during one week after the news release, and they concluded that the number of submission to Twitter about those topics increased by about $10 \%$ as compared to an ordinary week. The rate of users of Twitter in USA is about $1 / 4$ of all [46]. From this social experiment, the order of $v$ is estimated as $O(v)=1$ so that we set as $v=5$. The value of $v$, however does not so influence on our results.

\section{Results and Discussion}

\subsection{Time Behavior of Awareness}

Figure 5(a) shows the public awareness $\langle\xi\rangle$ as a function of time for the case of $\eta=3.0, t_{0}=15$ and $a_{0} \in[15,45]$. Although the calculation with those values of parameters may not precisely reproduce the observation, it can be said to explain the overall trends of that behavior. The results depend on $\eta$ only weakly. The calculation with $a_{0}=16$ shown here seems apparently incompatible to the observation, indicating the requirement for the other components of $\langle\xi\rangle$ to hold consistency. The strength of the cyber field for $a_{0}=16$ is $\Psi_{2}(t) \approx 0$ throughout the whole range of time, which means no contribution from the cyber world, whereas the other calculations in Figure 5(a) are not so.

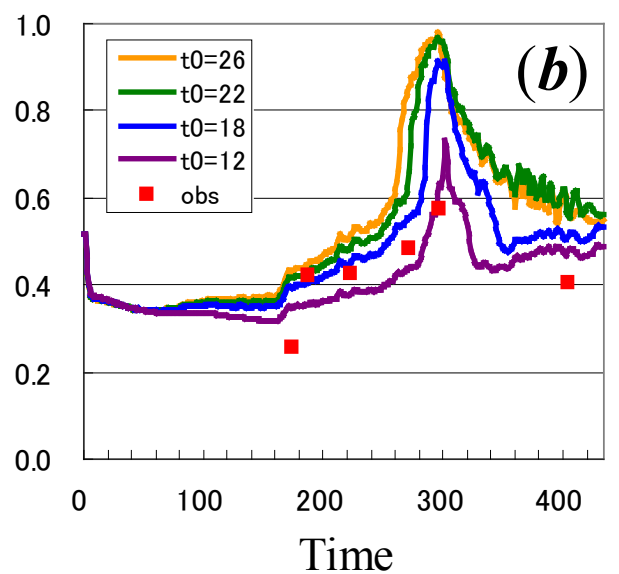

Figure 5. Comparison of the calculation $\langle\xi\rangle$ to the observation for the case of $\eta=3.0$. (a) $a_{0}$-dependence of calculation for the case of $t_{0=} 15$ and $a_{0} \in[16$, 45]. No bursts appear in the cyber world with $a_{0}=16$. (b) $t_{0}$-dependence of calculation for the case of $a_{0}=36$ and $t_{0} \in[12,26]$.

Figure 5(b) is the behavior of $<\xi>$ for the case of $a_{0}=36$, and $t_{0} \in[12,26]$. From these figures it becomes clear that the time $t_{\max }$ when the $\langle\xi\rangle$ becomes maximum varies as $t_{\max }=290 \sim 310$ depending on the values of those parameters. The time $t_{\max }$ is not necessarily the same value as the time $(t \approx 295)$ when the external information to be input into our model becomes maximum. The quantity $\langle\xi\rangle$ features a broad peak around $t=t_{\max }$ superimposed on a background that gradually increases with time. The appearance of this peak in $\langle\xi\rangle$ is due to the temporal strengthening of the cyber field, which will be discussed later.

Figure 6 shows an example for the time behavior of the distribution function $\Xi(\xi, t)$. The function $\Xi(\xi, t)$ scarcely depends on the initial condition after $t>50$. Although, only at around $t=t_{\max }$ (namely the time range when the $\langle\xi\rangle$ grows large), that function becomes to be one-sided towards a larger part of $\xi$ (as the curves at $\mathrm{t}=300$ and 325 in this figure), it broadly distributes throughout the whole region of $\xi$, with the 
gradual change of such a form with time.

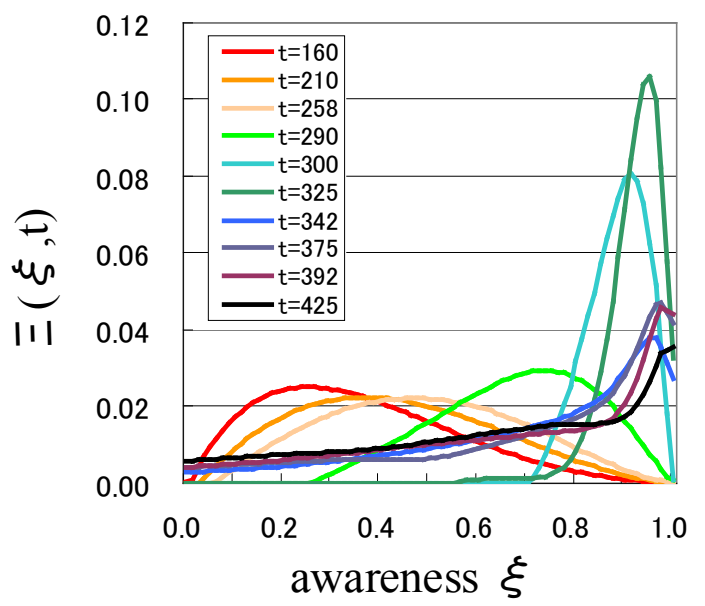

Figure 6. Time behavior of the distribution function $\Xi(\xi, t)$ for the case of $\eta=3.0, a_{0}=36$ and $t_{0}=22$.

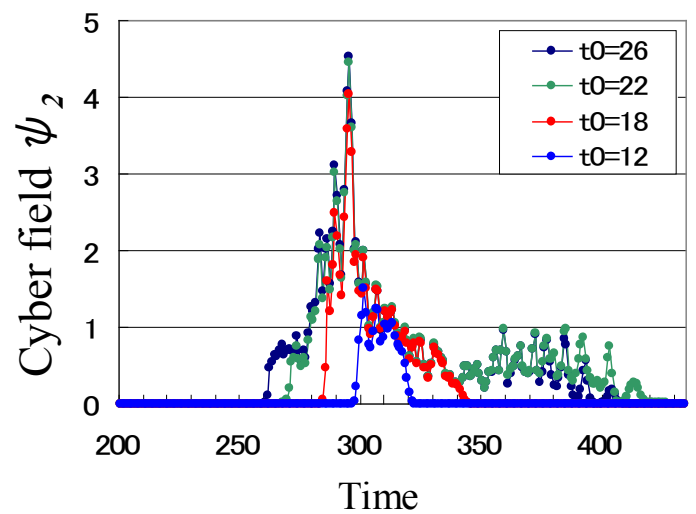

Figure 7. Time variation of the cyber field $\Psi 2(t)$ for the case of $\eta=3.0, a_{0}=36$ and $t_{0} \in[12,26]$.

Figure 7 shows the time variation of the amount of information in the cyber world, $\Psi_{2}(t)$. When the set of the parameters $\eta, a_{0}$ and $t_{0}$ is in a certain range of values that will be described later, the $\Psi_{2}(t)$ rapidly grows at $t \approx \tau_{\min }$ from a state of $\Psi_{2}\left(t<\tau_{\min }\right) \approx 0$, becomes to an almost saturated value, and rapidly decreases its value at $t=\tau_{\max }$ to become again $\Psi_{2}$ $\left(t>\tau_{\max }\right) \approx 0, \tau_{\min }$ and $\tau_{\max }$ being critical times determined by the parameters. This indicates the appearance of burst to transmit information in the cyber world. Clearly from (1), this is due to the occurrence of the positive feedback in the cyber world and to the temporal strengthening of the cyber field originated from instability. Such a situation corresponds to the public's strong seeking of information at around that time in the cyber world.

Since $\Psi_{2} \sim 0$ for an initial cyber field, it is required from (1) for the field to grow strongly that $O\left(\eta<\xi>{ }^{\mathrm{q}} \varepsilon \Psi_{1}\right) \rightarrow 1$, where the quantity $\varepsilon$, as described earlier, is the rate of the information in the real world, which is introduced in a cyber world, and is assumed to be a sufficiently small value $(\varepsilon=0.0001)$ so that it could be considered as a perturbation. To realize the burst in the cyber world, the following successive processes are to be occurred:

a) The increase of the amount of information in the real world $\Psi_{l}$.

b) The increase of $\langle\xi\rangle$ due to the former process (a), or to the increase of $\Psi_{2}$.

c) Further increase of the value of feedback, $\eta\langle\xi\rangle^{q}{ }_{\varepsilon} \Psi_{1}$, due to (a) and (b).

d) The increase of $\Psi_{2}$ due to (c).

e) The increase of the value of feedback, $\eta\left\langle\xi>^{q}\left(\Psi_{2}+\varepsilon \Psi_{l}\right)\right.$, due to $(d)$.

f) The increase of $\Psi_{2}$ due to (e).

g) The rapid growth of $\Psi_{2}$ by repeating the processes of $(\mathrm{f}) \rightarrow(\mathrm{b}) \rightarrow(\mathrm{e}) \rightarrow(\mathrm{f}) \rightarrow \ldots$, namely the realization of a burst.

On the other hand, the process to escape from the burst is:

(a') the appearance of the tangible effect of oblivion due to the elapse of time and the resultant decrease of $\langle\xi\rangle$.

(b') the decrease of the value of feedback due to the process (a').

(c') the decrease of $\Psi_{2}$ due to (b').

(d') the rapid decrease of the value of feedback below 1.0 and the disappear of the burst.

The parameters relating to the appearance and disappearance of the burst are $\eta, a_{0}$ and $t_{0}$ in our model. Figure 8 shows the curves of threshold to distinguish the region where the burst occurs from the other region on the $t_{0} a_{0}$-plane with $\eta$ as a variable parameter. There appear no bursts on the left hand side of the curves whereas they appear on the right hand side. The discontinuity at around $t_{0}=20$ seen in this figure is owed to the discontinuity of the input $\Psi_{1}$ around that time. So far as we discuss only in the region of $t_{0}<20$, the condition for the burst to occur corresponds to the following inequality region for our case of calculation;

$$
a_{0}>a_{\text {thresh }} \equiv(1091.0-191.4 \eta) \cdot t_{0}^{-1.09}
$$

The tipping point, that is the threshold for the beginning of burst, has been pointed out to widely exist in the region of economic and commercial activities together with the region of natural sciences [28]. The above equation (27) corresponds to the tipping point of our case. Under the condition of $\eta$ and $t_{0}$ in the region where they do not exceed the tipping point, the oblivion occurs too fast for the $\langle\xi\rangle$ to grow remarkably even if the $\Psi_{I}$ becomes large. In such a case it is considerably difficult to make fit the calculation to observation. When the $a_{0}$ is relatively stronger than the $t_{0}$ (that is the upper right part in Figure 8), the above unequal equation is satisfied so that the $\langle\xi\rangle$ becomes large with the increase of $\Psi_{1}$. When the $a_{0}$ becomes much larger than the $a_{\text {thresh }}$ given by (27), on the other hand, the $\langle\xi\rangle$ grows to continuously hold a large value for a long time so that its feature may no more be called as a burst. Our calculation with the assumption of burst gives results much better than the case without any bursts (see Figure 5(a)). This strongly indicates that the feature of $\langle\xi\rangle$ at around $t \approx t_{\max }$ is determined under the direct influence of the explosive transmission of information in the cyber world with the $\eta, t_{0}$ and $a_{0}$ of their respective values near the threshold given by (27). 


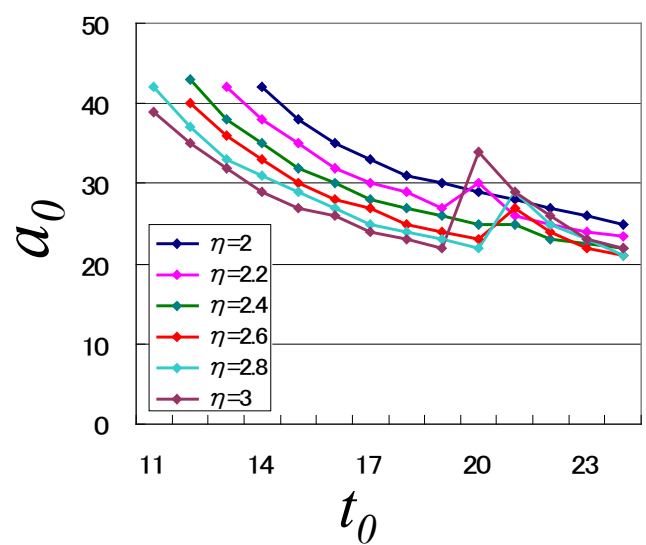

Figure 8. Threshold for the occurrence of burst. The discontinuity near $t_{0} \approx 21$ is due to the discontinuous trend in the input $\Psi_{I}(t)$.

\subsection{Burst in the Cyber World and Prevalence Phenomena}

As already described a critical condition for the occurrence of burst in the cyber world is $\eta^{\cdot}\left\langle\xi>^{q} \cdot \varepsilon \Psi_{I}=1\right.$. When the $<\xi>$ gradually increases with time or the $\Psi_{1}$ fluctuates toward the direction of its increase, such a condition for the bust may be accidentally held at some time. Once such a condition is held, the feedback continues after then with a feedback constant greater than 1 so that the burst of $\Psi_{2}$ also continues. In this case the burst starts absolutely in accident and spontaneously ceases when the oblivion makes the $\langle\xi\rangle$ decreased. To study the possible occurrence of such a case, we make here a trial calculation by taking the following input for the quantity $\Psi_{l}$.

$$
\Psi_{1}\left(t \geq t_{2}\right)=\Psi_{1}\left(t_{1}+r \cdot\left(t_{2}-t_{1}\right)\right)
$$

where $r$ is an uniform random number within a range $[0,1]$. The above equation means that the value of $\Psi_{l}$ at any time in $t \geq t_{2}$ is set equal to the $\Psi_{1}$ at a randomly selected time $t_{3} \equiv t_{1}+r \cdot\left(t_{2}-t_{l}\right)$. Namely the $\Psi_{1}$ at any time in $t \geq t_{2}$ is assumed to be given by the repeated but randomly selected $\Psi_{1}$ from the region $t \in\left[t_{1}, t_{2}\right]$.
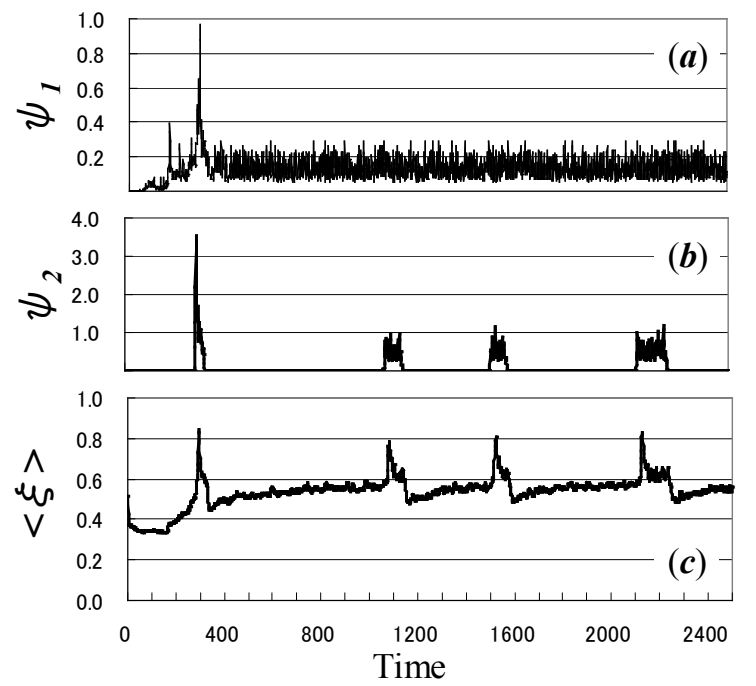

Figure 9. (a) The input $\Psi_{1}(t)$ which randomly varies in $t>t_{\max }=427$; (b) Time-varying cyber field $\Psi 2(t)$ for the case of $\eta=3.0, a_{0}=36$ and $t_{0}=15$; (c) Time behavior of $\langle\xi\rangle$ for the same case of $\eta, a_{0}$ and $t_{0}$ as (b).
Figure 9 (a)-(c) show the time behavior of $\Psi_{1}, \Psi_{2}$ and $<\xi>$ up to $t \sim 2500$ under the condition of $\left(\eta=3, a_{0}=35, t_{0}=15, t_{l}=320\right.$, $t_{2}=427$ ) for an example, where we can see four times of bursts in $\Psi_{2}$ and $\langle\xi\rangle$. The features of $\Psi_{2}$ and $\langle\xi\rangle$ indicate that the bursts spontaneously appear and disappear quasi-periodically even when the $\Psi_{l}$ has not a value as large as its maximum near $t \sim t_{\max }$. Such a phenomenon means the cyber world to be quasi-periodically excited, and it seemingly corresponds to the phenomenon of the quasi-periodic appearance of similar topics during a certain period of time in the SNS world [23]. Since the stimulus from the real field is of $\varepsilon=0.0001$ in our case, such a small input into the cyber field can be easily realized only if a minor fraction of information in the real world participates in it as a perturbation. Another example for the quasi-cyclic appearance of a certain topic in the cyber world is as follows.

Figure 10 shows the secular variation of the number of articles that can be retrieved from the Internet websites with a key word "Global Warming" in Japanese [47, 48]. The content of this database has been accumulated since 2002, especially after 2010 in earnest. Since the articles on websites are, in general, carried for a long time, the number of articles retrievable at a certain time corresponds almost to the number of total articles produced and accumulated up to the time. Hence the secular form of such a quantity has a tendency to increase gradually with time as seen in this figure. The increase of the number of articles after 2010 may be due somewhat to the growing use of the Internet owing to the spread of PC and smart phones. In fact the using rate of Internet in Japan was $70.8 \%$ in 2005 , and $78.2 \%$ at 2010 , but has grown to $92.0 \%$ in 2016 [43]. We should note in this figure that in addition to the background form of gradual growth with time, there appeared many spikes of the quantity in the past. This is due to the sudden increase of the pubic awareness in some special topics regarding GW, and hence to the burst of information supply which occurred quasi-periodically.

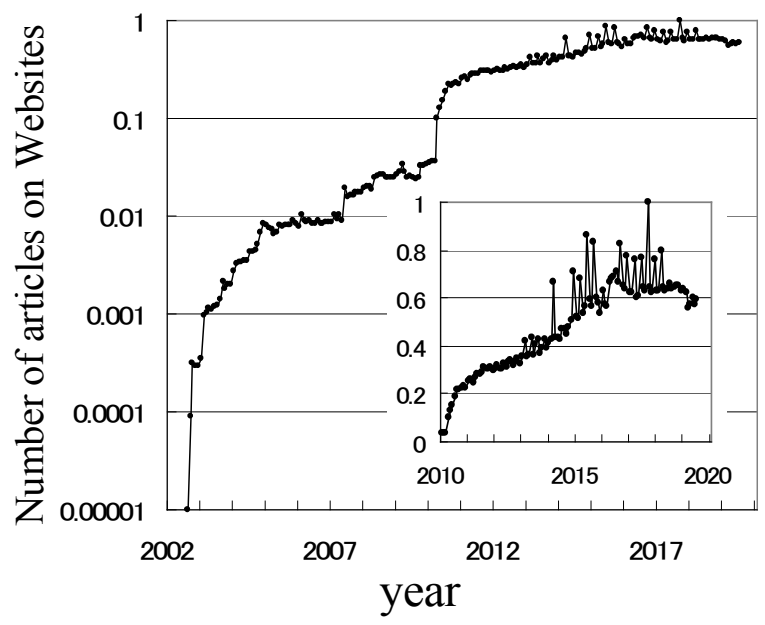

Figure 10. Number of Japanese articles on $G W$ that appeared in every month on Websites (normalized the maximum as 1.0), The gathering of the articles by Japan Diet Library became in earnest after 2010, the data for which is depicted in an inserted figure.

The way of understanding for the occurrence of burst can be 
applied to more general cases. According to the diffusion model of innovation [49], the characteristics of the public for accepting new innovation is conceptualized as shown in Figure 11(a), which is a distribution of the public's positiveness to adopt innovative commodities or ideologies. In order of positiveness, the public can be classified as (a) the innovators (about $2.5 \%$ of the total), (b) early adopters (13.5\%), (c) early majority (34\%), (d) late majority (34\%), and (e) laggards $(15 \%)$. When the innovator appears in the society, the early adopters, first of all, accepts the innovation. The remaining people, watching the innovators and early adopters, determine their own attitude whether or not they should accept the innovation. Here we classify the society of the innovators and the early adopters as the primary world, and the society of the remainder as the secondary world.

As shown in Figure 11(b), the interactions between these two worlds are the introduction of the innovation from the primary to the secondary worlds, and the observation and the imitation of the primary world by the people in the secondary world. The latter people, by observing the feature of their surroundings, feed it back to their own feature. When the repeated feedback of such imitation exceeds a critical value, the innovation diffuses throughout the followers and it becomes to be a prevalent phenomenon. When the people in the primary world forsake that innovation by adopting another type of innovation, the followers lose their ground to imitate so that the feedback gradually decreases finally to result in the end of the innovation. Thus the prevalent phenomena can be understood from the same ground as Figure (1) and (1) when we classify the people into two groups of society for the introducer of innovation and its adopter and consider the interacting duality of those societies. Thus the burst phenomenon including the prevalence, in general, can be treated as the interacting phenomenon taking place between the systems with the duality, by which methodology we can understand the epidemic diffusion, and the prevalence of attitude, thoughts and rumors [29-34].
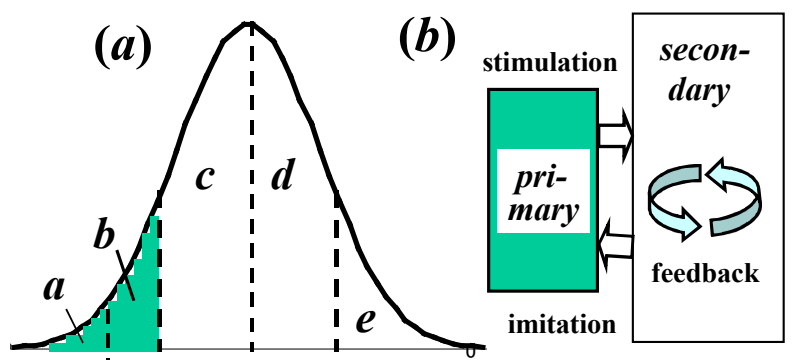

Figure 11. (a) Distribution of the acceptors of innovation, according to Rogers [49]. a: innovators, b: early adopters, c: early majority, d: late majority, e: laggards. (b) Its schematic diagram. As compared to Figure 1, the innovators correspond to the external events, the early adopters to the primary, real field, and the remainder to the secondary, cyber field.

\section{Conclusion}

Our model calculates the collective awareness of the public by using the field of information environment. The field is composed of two contributions from the real and cyber worlds.
The cyber world is assumed to relate to the real world by introducing the information from the real world by a certain definitive amount. The composite field from those worlds, together with the oblivion effect, makes the change of the public awareness. The duality of information field in the two worlds has revealed to have a characteristic such that the information released in the real world induces the burst of information transmission in the cyber world. Although it was not sure whether the rise of the awareness to GW in 2008 2009 in Japan is really owed to a contribution from the cyber field, we ascertained by introducing the dual model of information fields that the public awareness at that time can well be understood as to be influenced by the explosive cyber field.

Such an explosive appearance, that is, the burst of information field and its spontaneous cease occurs in the case when a certain function of the public awareness and the amount of information in the real world exceeds a critical condition, so that it is quite possible for the critical condition to be held suddenly by chance or to be held repeatedly. Such a phenomenon can be understood as a sudden appearance and sudden cease of prevalence. Thus the interactive dual system was found to lead quasi-periodic instability, that is the prevalence, in our society not only for the amount of information but for other innovative things, for instance, the demand of a sort of commodities, the thought, the epidemics, the rumor and so on.

\section{References}

[1] Statista, "News sources used in European countries in 2018", http://www.

statista.com/statistics/422687/news-sources-in-europen-countr ies/, Sept. 19, 2019 (retrieved).

[2] Pew Research Center, "More Americans get news often from social media than print paper", https://www.pewresearch.org/fact-tank/2018/12/10/social_media_o utpaces_print_newspapers_in_the_U.S._as_a_news_source", Sept. 19,2019 (retrieved).

[3] Japan Press Research Institute, National polls on the news media, 1st 10th, (2018) (in Japanese).

[4] NTT Docomo Mobile Society Research Institute, Usage trends of smart and mobile phones deciphered by the data 2018-2019 (Mobile phone social white paper), Chuoukeizaisha, Tokyo, 2018 (in Japanese).

[5] F. Schweitzer and J. A. Holyst, "Modelling collective opinion formation by means of active Brownian particles," Eur. Phys. J, B15, 723-732, 2000.

[6] F. Gargiulo and S. Huet, "Opionion dynamics in a group-based society," European Physics Letters, 91, 58004, 2010 .

[7] A. Jedrzejewski and K. Sznajd-Weron, "Impact of memory on opinion dynamics," Physica, A505, 306-315, 2018.

[8] D. Helbing, "A mathematical model for the behavior of individuals in a social field," J. Math. Soc. 19, 189-219, 1994. 
[9] J. Neirotti, "Consensus formation times in anisotropic societies," Phys. Rev., E95, 062305, 2017.

[10] C. Castellano, S. Fortunato and V. Loreto, "Statistical physics of social dynamics," Rev. Mod. Phys., 81, 591-646, 2009.

[11] A. H. Rodriguez and Y. Moreno, "Effect of mass media action on the Axlrod model with social influence," Phys. Rev., E82, 016111, 2010.

[12] B. Yun and Y. C. Cho, "Analyzing the effectiveness of public policy advertising on attitude and behavior change," J. Bus. Econ. Res., 12, 357-370, 2014.

[13] M. Pineda and G. M. Buendia, "Mass media and heterogeneous bounds of confidence in continuous opinion dynamics," Physica, A420, 73-84, 2015.

[14] K. Fan and W. Pedrycz, "Evolution of public opinions in closed societies influenced by broadcast media," Physica, A 472, 53-66, 2017.

[15] L. Deng, Y. Liu and Q-A. Zeng, "How information influences on individual opinion evolution," Physica, A 391, 6409-6417, 2012.

[16] J. R. Zaller, The nature and origins of mass opinion, Cambridge Univ. Press, NY, USA, 1992.

[17] T. Ohnishi, "A collective model for the formation of public opinion: an application to nuclear public acceptance", Mathl. Comput. Modelling, 19, 95-111, 1994.

[18] T. Ohnishi, "A mathematical model of the activities for public acceptance and the resultant reaction of the public: an application to the nuclear problem," Mathl. Comput. Modelling, 21, 1-30, 1995.

[19] K. Kulakowski, "Opinion polarization in Receipt-Accept-Sample model,” Physica, A388, 469-476, 2009.

[20] S. Biswas, A. Chatterjee and P. Sen, "Disorder induced phase transition in kinetic models of opinion dynamics," Physica, A391, 3257-3265, 2012.

[21] T. Ohnishi and K. Shimano, "Public interest immersed in the field of information environment: how has Japanese interest in energy and environmental problems varied?" Reports in Advances of Physical Sciences, 2, 1850005, 2018.

[22] L. Weng, A. Flammini, A. Vespignani and F. Menczer, "Competition among memes in a world with limited attention," Science Reports, 2, 335, 2012.

[23] J. Leskovec, L. Backstrom amd J. Kleinberg, "Meme-tracking and the Dynamics of the News Cycle," Proceedings 15th ACM SIGKDD Intern. Conf. Knowledge Discovery Data Mining, Paris, June, 2009.

[24] M. Gentzkow, "Small media, big impact," Science, 358, 726-727, 2017.

[25] D. Centola, J. Becker, D. Brackbill and A. Baronchelli, "Experimental evidence for tipping points in social convention," Science, 360, 1116-1119, 2018.

[26] C. Doyle, B. K. Szymanski and G. Korniss, "Effects of communication burstiness on consensus formation and tipping points in social dynamics," Phys. Rev., E95, 062303, 2017.

[27] S. Pinto, F. Albanese, C. O. Dorso and P. Balenzuela,
"Quantifying time-dependent media agenda and public opinion by topic modeling," Physica, A524, 614-624, 2019.

[28] M. Gladwell, The tipping point: how little things can make a big difference, Little, Brown and Company, Boston, 2000.

[29] C. Granell, S. Gomez and A. Arenas, "On the dynamical interplay between awareness and epidemics spreading in multiplex networks," Phys. Rev. Lett., 111, 128701, 2013.

[30] R. Wang and Q. A. Wang, "Dual modeling of political opinion networks," Phys. Rev., E84, 036108, 2011.

[31] X. Dong, Y. Liu, C. Wu, Y. Lian and D. Tang, "A double-identity rumor spreading model," Physica, A528, 121479, 2019.

[32] Y. Yi, Z. Zhang and C. Gan, "The outbreak threshold of information diffusion over social-physical networks," Physica, A526, 121128, 2019.

[33] C. Wang, G. Wang, X. Luo and H. Li, "Modeling rumor propagation and mitigation across multiple social networks," Physica, A535, 122240, 2019.

[34] ) H-W. Lee, N. Malik, F. Shi and P. Mucha, "Social clustering in epidemic spread on coevolving networks," Phys. Rev., E99, 062301, 2019.

[35] J. T. Ripberger, "Capturing curiosity: Using Internet search trends to measure public attentiveness," Policy Studies Journal, 39, 239-259, 2011.

[36] L. Guggenheim, S. M. Jang, S. Y. Bae and W. R. Neuman, "The dynamics of issue frame competition in traditional and social media," Annals, AAPSS, 659, 207-224, 2015.

[37] T. L. Milfont, "The interplay between knowledge, perceived efficacy, and concern about global warming and climate change: A one-year longitudinal study," Risk Analysis, 32, 1003-1020, 2012.

[38] A. Malka,. A. Krosnick and G. Langer, "The association of knowledge with concern about global warning: Trusted information sources shape public thinking," Risk Analysis, 29, 633-647, 2009.

[39] T. Ohnishi, "A mathematical model for the occurrence of historical events,” J. Phys.: Conf. Ser., 936, 012024, 2017.

[40] NHK Archives, https://www.nhk.or.jp/archives/document/, Sept. 5, 2019 (retrieved in Japanese).

[41] Oya Soichi Library Magazine Article Index Search, "Web OYA-bunko", http://www.oya-bunko,or.jp/, Aug. 16, 2019 (retrieved in Japanese).

[42] Google Trends, http;//www.google.com/trends, and Google Insights, http:/www.google.com/insights/search/, Aug. 16, 2019 (retrieved).

[43] United Nations, Statistical Yearbook: sixty-first issue, New York, 2018.

[44] National Institute of Environmental Studies, Japan, Report of public opinion survey on environmental awareness, Tokyo, 2016 (in Japanese).

[45] G. King, B. Schneer and A. White, "How the news media activate public expression and influence national agendas," Science, 358, 776-780, 2017. 
[46] Pew Research Center, "Social Media Update 2016," https://www.pewinter net.org/2016/11/11/social-media-update-2016/, Aug. 30, 2019 (retrieved).

[47] National Diet Library, Japan, "Database WARP," http://warp.da.ndl.go.jp/, Aug. 29, 2019 (retrieved in Japanese).
[48] T. Kimezawa and Y, Murayama, "Preservation of database on website-accessibility survey for Dnavi and sustainability of online databases," J. Inf. Sci. Tech. Assoc., Japan, 67, 459-464, 2017 (in Japanese).

[49] E. Rogers, Diffusion of Innovation, Free Press, New York: 1995. 